\title{
Kybernetika
}

\section{Jan Voříšek}

Estimation and bimodality testing in the cusp model

Kybernetika, Vol. 54 (2018), No. 4, 798-814

Persistent URL: http://dml.cz/dmlcz/147425

\section{Terms of use:}

(C) Institute of Information Theory and Automation AS CR, 2018

Institute of Mathematics of the Czech Academy of Sciences provides access to digitized documents strictly for personal use. Each copy of any part of this document must contain these Terms of use.

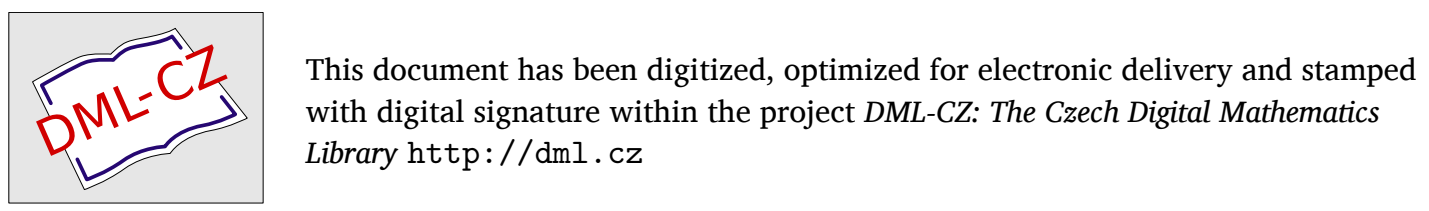




\title{
ESTIMATION AND BIMODALITY TESTING IN THE CUSP MODEL
}

\author{
JAN VOŘÍŠEK
}

The probability density function of the stochastic cusp model belongs to the class of generalized exponential distributions. It accommodates variable skewness, kurtosis, and bimodality. A statistical test for bimodality of the stochastic cusp model using the maximum likelihood estimation and delta method for Cardan's discriminant is introduced in this paper, as is a necessary condition for bimodality, which can be used for simplified testing to reject bimodality. Numerical maximum likelihood estimation of the cusp model is simplified by analytical reduction of the parameter space dimension, and connection to the method of moment estimates is shown. A simulation study is used to determine the size and power of the proposed tests and to compare pertinence among different tests for various parameter settings.

Keywords: multimodal distributions, cusp model, bimodality test, reduced maximum likelihood estimation

Classification: $62 \mathrm{~F} 03$

\section{INTRODUCTION}

The cusp model was given its name thanks to classification of singularities by Vladimir Arnold [1] within deterministic models of catastrophe theory proposed by Rene Thom [17. Theory of the stochastic version was established by Lauren Cobb and his colleagues (4, [6], Cobb and Watson [5], Cobb, Koppstein, and Chen [7]), who classified a stochastic cusp model within the class of multimodal distributions from the exponential family and employed the method of moments and the maximum likelihood approach for estimation of its parameters. There are other methods for estimation in catastrophe models; however, the maximum likelihood approach was found to be the most appealing by Grasman, van der Maas and Wagenmakers ([11]), who implemented the maximum likelihood method into the cusp package for the statistical computing environment $\mathrm{R}$. It allows the state variable and parameters of the cusp model to be given as a multivariate approximation of the measured variables. Flexibility of the cusp model was utilized in various papers (e.g., Creedy and Martin [8, Fernandes [10, Koh, Fong and Chan 14, Barunik and Vosvrda [3], Barunik and Kukacka [2], and Diks and Wang [9]) dealing with crashes on financial markets.

DOI: $10.14736 /$ kyb-2018-4-0798 
Bimodality of the probability density function of the cusp model can be determined by the sign of Cardan's discriminant, which is negative when the probability density function has two modes, and nonnegative in the unimodal case. This property is used to inspect the probability of crash in some papers, but the distribution of Cardan's discriminant remains mostly unaddressed. 11

This paper approximates the distribution of Cardan's discriminant using the delta method; its asymptotic distribution is used for statistical testing where the null hypothesis is rejected if there is sufficient statistical evidence against bimodality of the distribution. Bimodality could sometimes be rejected with the aid of a simpler test that utilizes a necessary condition for the negativity of Cardan's discriminant. The size and power of these tests are determined using a simulation study and compared with the one sided likelihood ratio test for Cardan's discriminant. A test of unimodality for general distribution called dip (by Hartigan and Hartigan [12]) is compared to the delta method approach and the likelihood ratio test with the opposite null and alternative hypothesis for the testing of unimodality in the cusp model.

The standard approach to the maximum likelihood estimation of the cusp model parameters numerically optimizes all parameters of the model. This paper shows a reduction of the parameter space by two dimensions, and connects this result to parameter space reduction by the method of moments described by Matz ([16]).

The rest of the paper is organized as follows. Section 2.1 describes the cusp model and its connection to the multimodal distributions; Section 2.2 shows different approaches to the estimation of parameters and a simplified estimation procedure; and the statistical tests of bimodality are proposed in Section 3.1. Section 3.2 utilizes the methodology for estimating simulated results and determining the size and power of the statistical tests. Section 4 summarizes the results and concludes the paper.

\section{CUSP MODEL}

\subsection{Multimodal distributions}

This section recapitulates the connection of the stochastic cusp model to the exponential class of multimodal distributions. The standard parameterization within both approaches is shown, as well as the transformation from one to the other following Cobb et al. [7.

The generalized exponential family of distributions is characterized by the probability density function

$$
f_{k}(x)=\xi_{k}(\mathbf{b}) \exp \left[\int_{l}^{x} \frac{g(s)}{v(s)} \mathrm{d} s\right],
$$

where $g(x)=\sum_{i=0}^{k} b_{i} x^{k}$ is a polynomial function of order $k>0$, and function $v(x)$ has one of the following forms:

\footnotetext{
${ }^{1}$ Fernandes [10] used simulations to determine a 90\% confidence interval for Cardan's discriminant by bootstrapping.
} 


$$
\begin{array}{rlr}
\text { typeN : } & v(x)=1 & -\infty<x<\infty, \\
\text { typeG : } & v(x)=x & 0<x<\infty, \\
\text { typeI : } & v(x)=x^{2} & 0<x<\infty, \\
\text { typeB : } & v(x)=x(1-x) & 0<x<1 .
\end{array}
$$

Let $(l, u)$ be an open interval, where $v(x)$ is positive and $\xi_{k}(\mathbf{b})$ is a normalizing constant such that $\int_{l}^{u} f_{k}(x) \mathrm{d} x$ equals one.

Consider a cubic polynomial

$$
g(x)=b_{0}+b_{1} x+b_{2} x^{2}+b_{3} x^{3},
$$

where $b_{3}<0$; given a type $N$ scaling function $v(x)=1$, it yields the following probability density function ${ }^{2}$

$$
f_{3}(x)=\xi_{3}(\mathbf{b}) \exp \left[b_{0} x+\frac{b_{1}}{2} x^{2}+\frac{b_{2}}{3} x^{3}+\frac{b_{3}}{4} x^{4}\right],
$$

where $\xi_{3}(\mathbf{b})$ is a normalizing constant depending on parameters $b_{0}, b_{1}, b_{2}$, and $b_{3}$. Transformation of parameters

$$
\begin{aligned}
& \sigma=\left(-b_{3}\right)^{-1 / 4}, \\
& \lambda=-b_{2} /\left(3 b_{3}\right), \\
& \beta=\left(b_{1}+b_{2} \lambda\right) \sigma^{2}, \\
& \alpha=\sigma g(\lambda),
\end{aligned}
$$

yields probability density function

$$
f_{\theta}(z)=\xi(\theta) \exp \left[\alpha z+\frac{\beta}{2} z^{2}-\frac{1}{4} z^{4}\right],
$$

where $z=\frac{x-\lambda}{\sigma}, \xi(\theta)$ is a normalizing constant, and $\theta$ stands for a vector of parameters $(\alpha, \beta, \lambda, \sigma)^{T}$. This probability density function is known as a cusp distribution and its parameterization is particularly useful for identifying the bimodality (or unimodality) of the distribution using Cardan's discriminant

$$
\delta_{C}=\left(\frac{\alpha}{2}\right)^{2}-\left(\frac{\beta}{3}\right)^{3}
$$

which is negative when the probability density function is bimodal and non-negative in the unimodal case. The parameters $\alpha$ (asymmetry) and $\beta$ (bifurcation) are invariant with respect to changes in $\lambda$ (location) and $\sigma$ (scale) and they have the following approximate interpretations. If $\delta_{C} \geq 0$ then $\alpha$ measures the skewness, and $\beta$ the kurtosis; likewise, when $\delta_{C}<0$ then $\alpha$ indicates the relative height of the two modes, and $\beta$ their relative separation.

\footnotetext{
${ }^{2}$ Called a quartic exponential distribution $([16])$
} 


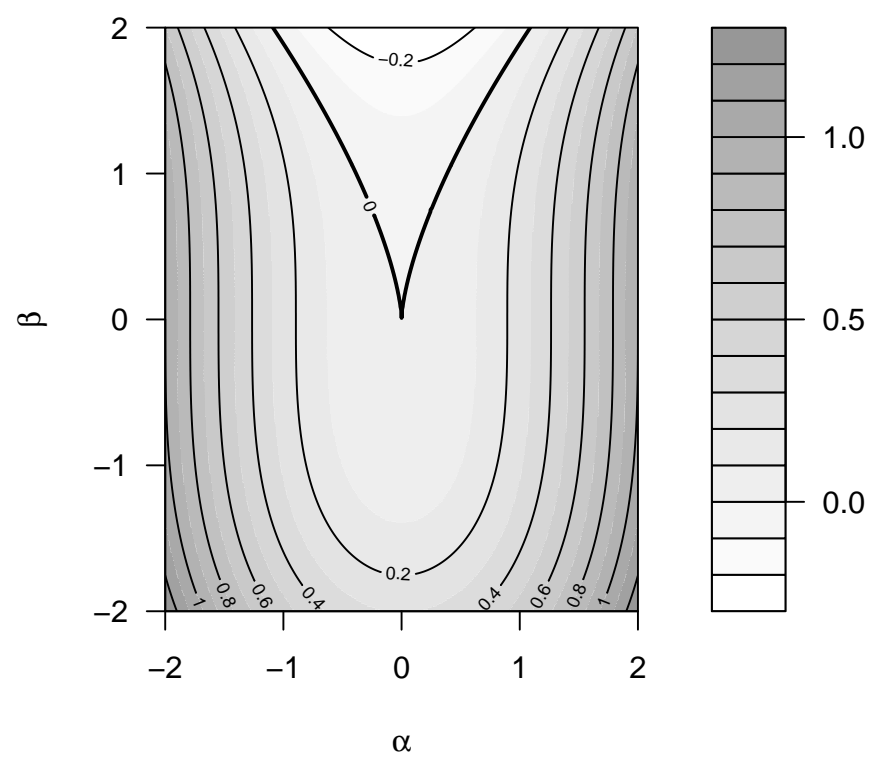

Fig. 1. Contour plot of Cardan's discriminant for $\alpha \in(-2,2)$ and $\beta \in(-2,2)$.

\subsection{Reduced estimation}

Different estimation techniques can be used in the cusp model (6) for the parameters $\theta=(\alpha, \beta, \lambda, \sigma)^{T}$. The maximum likelihood approach implemented in the $\mathrm{R}$ package cusp ([1]) allows for parameters $\alpha$ and $\beta$ to be time-varying with the aid of a linear dependence on independent variables $\zeta_{1}, \ldots, \zeta_{q}$.

$$
\begin{aligned}
& \alpha(t)=\alpha_{0}+\alpha_{1} \zeta_{1 t}+\ldots+\alpha_{q} \zeta_{q t}, \\
& \beta(t)=\beta_{0}+\beta_{1} \zeta_{1 t}+\ldots+\beta_{q} \zeta_{q t},
\end{aligned}
$$

Numerical maximization of the likelihood function is optimized with respect to all parameters; it means four dimensions in the case, where values $\alpha$ and $\beta$ are constant. Given a sample of i.i.d. observations $x_{i}$, the log-likelihood function of the cusp model with constant parameters stems from the general probability density function (6) as

$$
\begin{aligned}
l l_{n}(\theta)= & \sum_{i=1}^{n} \alpha \frac{x_{i}-\lambda}{\sigma}+\frac{\beta}{2}\left(\frac{x_{i}-\lambda}{\sigma}\right)^{2}-\frac{1}{4}\left(\frac{x_{i}-\lambda}{\sigma}\right)^{4} \\
& -n \log \left(\sigma \int_{-\infty}^{\infty} \exp \left[\alpha z+\frac{\beta}{2} z^{2}-\frac{1}{4} z^{4}\right] \mathrm{d} z\right)
\end{aligned}
$$

where the normalizing constant is simplified using substitution $z=(x-\lambda) / \sigma$. Estimation 
of four parameters can be reduced by differentiating the log-likelihood function with respect to $\lambda$ and $\sigma$ :

$$
\begin{aligned}
\frac{\partial}{\partial \lambda} l l_{n}(\theta) & =-n \frac{\alpha+\beta \bar{z}-\overline{z^{3}}}{\sigma}, \\
\frac{\partial}{\partial \sigma} l l_{n}(\theta) & =-n \frac{1+\alpha \bar{z}+\beta \overline{z^{2}}-\overline{z^{4}}}{\sigma},
\end{aligned}
$$

where $z_{i}=\left(x_{i}-\lambda\right) / \sigma$ and $\overline{z^{j}}=1 / n \sum_{i=1}^{n} z_{i}^{j}$, and laying both differences equal to zero yields:

$$
\begin{aligned}
& \hat{\beta}=\frac{\overline{z^{4}}-\overline{z^{3}} \bar{z}-1}{\overline{z^{2}}-\bar{z}^{2}}, \\
& \hat{\alpha}=\overline{z^{3}}-\hat{\beta} \bar{z}=-\frac{\overline{z^{4}} \bar{z}-\overline{z^{3}} \overline{z^{2}}-\bar{z}}{\overline{z^{2}}-\bar{z}^{2}} .
\end{aligned}
$$

Note that both estimates $\hat{\alpha}$ and $\hat{\beta}$ depend on $\lambda$ and $\sigma$ hidden in $\overline{z^{j}}$. Substituting expressions (14) and (13) into the log-likelihood function 10 allows us to numerically search for the optimum in merely two dimensions. Moreover, if $\alpha=0$, the probability density function is symmetric, which enables us to express the maximum likelihood estimator $\hat{\lambda}=\bar{x}$ since $\int_{-\infty}^{\infty} z \exp \left[\frac{\beta}{2} z^{2}-\frac{1}{4} z^{4}\right] \mathrm{d} z=0$ and only the estimator $\hat{\sigma}$ has to be found numerically.

A similar reduction of the polynomial form (4) was carried out by Matz [16. He employs the same property as Cobb et al. [7] when proposing moment recursion relations which connect $k+1$ parameters of the probability density function 11 to the first $2 k$ moments, namely:

$$
E\left(X^{j} g(X)\right)=E\left(j X^{j-1}\right)
$$

which implies:

$$
\sum_{i=0}^{3} b_{i} \mu_{m+i}=m \mu_{m-1},
$$

for $m=0, \ldots, 3$, where $\mu_{m}$ denotes the moments of the distribution (4). Using the first two equations $(m=0,1)$ one can express parameters $b_{0}$ and $b_{1}$ for given $b_{2}$ and $b_{3}$ and it can, by substitution (5) and basic algebraic operations, be shown that reduced estimates $\hat{\alpha}$ and $\hat{\beta} 14$ and 13 are equal within both approaches.

From the theory of exponential families (e.g., Lehmann and Romano [15]) we know that the maximum likelihood estimator exists and is unique; the usual initial points for numerical optimization are the moment estimates (by Cobb et al. 7]). Ratio of the number of iterations ${ }^{3}$ is almost four times in favor of the reduced estimation (see Table 1); the median numbers of iterations are 63 and 227, respectively, for $10^{4}$ replications

\footnotetext{
${ }^{3}$ For numerical optimization of restricted and unrestricted parameter space was used optim function with default settings from $\mathrm{R}$ statistical software, version 3.3.3 .
} 


\begin{tabular}{lcc}
\hline est. par. & 2 & 4 \\
\hline median & 63 & 227 \\
mean & 63.3 & 237.3 \\
st.dev. & 7.9 & 58.2 \\
skew. & 4.7 & 1 \\
kurt. & 43.3 & 4.3 \\
\hline est. time & 0.06 & 0.09 \\
\hline
\end{tabular}

Tab. 1. Characteristics of number of iterations, and the average estimation time in seconds for two- and four- parameter estimation, sample size $10^{3}$, and $10^{4}$ replications.

and a sample size of $10^{3}$, where the estimation time in the reduced parameter space is about two-thirds of that achieved by the full parameter space estimation.

The dimension reduction by two is also attainable by analogous approach for timevarying parameters $\alpha(t)$ and $\beta(t)$. The log-likelihood function is extended into

$$
\begin{aligned}
l l_{\zeta}(\theta)=\sum_{i=1}^{n} & {\left[\left(\alpha_{0}+\alpha_{1} \zeta_{i}\right) \frac{x_{i}-\lambda}{\sigma}+\frac{\beta_{0}+\beta_{1} \zeta_{i}}{2}\left(\frac{x_{i}-\lambda}{\sigma}\right)^{2}-\frac{1}{4}\left(\frac{x_{i}-\lambda}{\sigma}\right)^{4}\right.} \\
& \left.-\log \left(\sigma \int_{-\infty}^{\infty} \exp \left[\left(\alpha_{0}+\alpha_{1} \zeta_{i}\right) \mathrm{d} z+\frac{\beta_{0}+\beta_{1} \zeta_{i}}{2} z^{2}-\frac{1}{4} z^{4}\right] \mathrm{d} z\right)\right],
\end{aligned}
$$

where terms with $\zeta_{i}$ could be perceived vectorwise. Differentiating log-likelihood function (17) with respect to $\lambda$ and $\sigma$ yields

$$
\begin{aligned}
& \frac{\partial}{\partial \lambda} l l_{n}(\theta)=-\frac{n}{\sigma}\left(\alpha_{0}+\alpha_{1} \bar{\zeta}+\beta_{0} \bar{z}+\beta_{1} \overline{\zeta z}-\overline{z^{3}}\right) \\
& \frac{\partial}{\partial \sigma} l l_{n}(\theta)=-\frac{n}{\sigma}\left(1+\alpha_{0} \bar{z}+\alpha_{1} \overline{\zeta z}+\beta_{0} \overline{z^{2}}+\beta_{1} \overline{\zeta z^{2}}-\overline{z^{4}}\right)
\end{aligned}
$$

where $\overline{\zeta z^{j}}=1 / n \sum_{i=1}^{n} \zeta_{i} z_{i}^{j}$ and laying 18 and 19 down to zero brings expression of level parameters

$$
\begin{aligned}
& \hat{\beta}_{0}=\frac{\overline{z^{4}}-\overline{z^{3}} \bar{z}-1-\beta_{1}\left(\overline{\zeta z^{2}}-\overline{\zeta z} \bar{z}\right)-\alpha_{1}(\overline{\zeta z}-\bar{\zeta} \bar{z})}{\overline{z^{2}}-\bar{z}^{2}}, \\
& \hat{\alpha}_{0}=-\frac{\overline{z^{4}} \bar{z}-\overline{z^{3}} \overline{z^{2}}-\bar{z}-\beta_{1}\left(\overline{\zeta z^{2}} \bar{z}-\overline{\zeta z} \bar{z}^{2}\right)-\alpha_{1}\left(\overline{\zeta z} \bar{z}-\bar{\zeta} \bar{z}^{2}\right)}{\overline{z^{2}}-\bar{z}^{2}} .
\end{aligned}
$$




\section{TESTING BIMODALITY}

\subsection{Formulation of the tests}

The positivity of parameter $\beta$ is a necessary condition for Cardan's discriminant (7) to be negative. This condition could be statistically tested at first using an asymptotic maximum likelihood estimator, $\sqrt{n}\left(\hat{\beta}-\beta_{0}\right) \stackrel{d}{\rightarrow} N\left(0, j\left(\theta_{0}\right)_{\beta}^{-1}\right)$, where $j(\theta)_{\beta}^{-1}$ denotes the diagonal element of the inverted Fisher information matrix $j(\theta)$ corresponding to $\beta$. The null hypothesis of the beta-test would be $H_{0}: \beta \geq 0$ with a one-sided alternative $H_{1}: \beta<$ 0 and the corresponding test statistics $\sqrt{n} \hat{\beta} / \sqrt{\hat{j}(\hat{\theta})_{\beta}^{-1}}$ has an asymptotically standard normal distribution for $\beta=0$. Rejection of the null hypothesis implies rejection of the negativity of Cardan's discriminant, which in its turn means rejection of bimodality.

For testing bimodality while taking the value of $\alpha$ into account, we can use an approximate distribution of Cardan's discriminant derived using the delta method, which yields:

$$
\sqrt{n}\left(\hat{\delta}_{C}-\delta_{0}\right) \stackrel{d}{\rightarrow} N\left(0, \nabla h\left(\theta_{\mathbf{0}}\right)^{T} j\left(\theta_{0}\right)^{-1} \nabla h\left(\theta_{\mathbf{0}}\right)\right),
$$

where function $h(\theta)=\left(\alpha^{2} / 4-\beta^{3} / 27\right)$ transforms the parameters of the cusp probability density function (6) into Cardan's discriminant, and $\nabla h(\theta)=\frac{\partial}{\partial \theta} h(\theta)$. This result allows us to propose a delta-test for bimodality, where we would like to test $H_{0}: \delta_{C} \leq 0$ against $H_{1}: \delta_{C}>0$ and the test statistic of the delta-test

$$
\sqrt{n} \frac{\hat{\delta}_{C}}{\sqrt{\nabla h(\hat{\theta})^{T} \hat{j}(\hat{\theta})^{-1} \nabla h\left(\hat{\theta_{0}}\right)}}
$$

has an asymptotically normal distribution for $\delta_{C}=0$ and $\alpha \neq 0$ which implies $\beta \neq 0$. Rejection of the null hypothesis means rejection of bimodality similar to the beta-test case. For $\alpha=0$ and $\beta=0$, it is possible to derive the asymptotic distribution using second order Taylor expansion, which yields chi-square distribution of the test statistics; $4 n\left(\hat{\delta}_{C}\right) / \hat{j}(\hat{\theta})_{\alpha}^{-1}$.

A third approach to testing bimodality is the likelihood ratio test, with the parameter space reduced by

$$
\beta \geq 3\left(\frac{\alpha}{2}\right)^{2 / 3}
$$

under null hypothesis $H_{0}: \delta_{C} \leq 0$ against alternative $H_{1}: \delta_{C}>0$. Test statistics $2\left(l l_{n}(\hat{\theta})-l l_{n}(\tilde{\theta})\right)$ has an asymptotically $\frac{1}{2}\left(\chi_{1}^{2}+\chi_{0}^{2}\right)$ distribution 4 under the null hypothesis, where $\tilde{\theta}$ stands for the parameters estimated under the restriction 24.

The general test of unimodality called dip is a natural candidate for comparison with the proposed tests. The dip test (by Hartigan and Hartigan [12]) tests unimodality of a general distribution, where the dip statistic is the maximum difference between empirical distribution function, and the unimodal distribution function that minimizes

\footnotetext{
${ }^{4}$ Given by equality of lower and upper bound of the distribution ( see Kodde and Palm [13]).
} 
that maximum difference. 5 Tested hypothesis of the delta-test and likelihood ratio test has to be reversed into $H_{0}: \delta_{C} \geq 0$ and $H_{1}: \delta_{C}<0$ to be comparable with the dip test.

For inference on the Fisher information matrix, let us define

$$
\iota_{p}(\theta)=\int_{-\infty}^{\infty} z^{p} \exp \left[\alpha z+\frac{\beta}{2} z^{2}-\frac{1}{4} z^{4}\right] \mathrm{d} z .
$$

Then the elements of the Fisher information matrix are given as

$$
\begin{aligned}
-E\left(\frac{\partial^{2}}{\partial \alpha^{2}} \log \left(f_{\theta}(x)\right)\right) & =\frac{\iota_{0}(\theta) \iota_{2}(\theta)-\iota_{1}(\theta)^{2}}{\iota_{0}(\theta)^{2}} \\
-E\left(\frac{\partial^{2}}{\partial \alpha \partial \beta} \log \left(f_{\theta}(x)\right)\right) & =\frac{\iota_{0}(\theta) \iota_{3}(\theta)-\iota_{1}(\theta) \iota_{2}(\theta)}{2 \iota_{0}(\theta)^{2}}, \\
-E\left(\frac{\partial^{2}}{\partial \alpha \partial \lambda} \log \left(f_{\theta}(x)\right)\right) & =\frac{1}{\sigma} \\
-E\left(\frac{\partial^{2}}{\partial \alpha \partial \sigma} \log \left(f_{\theta}(x)\right)\right) & =\frac{1}{\sigma} \frac{\iota_{1}(\theta)}{\iota_{0}(\theta)} \\
-E\left(\frac{\partial^{2}}{\partial \beta^{2}} \log \left(f_{\theta}(x)\right)\right) & =\frac{\iota_{0}(\theta) \iota_{4}(\theta)-\iota_{2}(\theta)^{2}}{4 \iota_{0}(\theta)^{2}} \\
-E\left(\frac{\partial^{2}}{\partial \beta \partial \lambda} \log \left(f_{\theta}(x)\right)\right) & =\frac{1}{\sigma} \frac{\iota_{1}(\theta)}{\iota_{0}(\theta)} \\
-E\left(\frac{\partial^{2}}{\partial \beta \partial \sigma} \log \left(f_{\theta}(x)\right)\right) & =\frac{1}{\sigma} \frac{\iota_{2}(\theta)}{\iota_{0}(\theta)}, \\
-E\left(\frac{\partial^{2}}{\partial \lambda^{2}} \log \left(f_{\theta}(x)\right)\right) & =-\frac{1}{\sigma^{2}} \frac{\beta \iota_{0}(\theta)-3 \iota_{2}(\theta)}{\iota_{0}(\theta)}, \\
-E\left(\frac{\partial^{2}}{\partial \lambda \partial \sigma} \log \left(f_{\theta}(x)\right)\right) & =-\frac{1}{\sigma^{2}} \frac{\alpha \iota_{0}(\theta)+2 \beta \iota_{1}(\theta)-4 \iota_{3}(\theta)}{\iota_{0}(\theta)} \\
-E\left(\frac{\partial^{2}}{\partial \sigma^{2}} \log \left(f_{\theta}(x)\right)\right) & =-\frac{1}{\sigma^{2}} \frac{\iota_{0}(\theta)+2 \alpha \iota_{1}(\theta)+3 \beta \iota_{2}(\theta)-5 \iota_{4}(\theta)}{\iota_{0}(\theta)} .
\end{aligned}
$$

\subsection{Simulation results}

To determine the power and size of the proposed tests, a simulation study was performed for different combinations of parameters $\alpha$ and $\beta$ and $10^{3}$ replications. The least favorable properties of the delta-test lies in its low power in the neighborhood of $\alpha=0$ (see Table 2), and lowering power for $\beta$ decreasing away from zero (compare Table 7 and Table 8 for $10^{3}$ sample size, caused by inflation of variance of the delta method distribution.

From the symmetry guaranteed by $\alpha=0$, the expected values of odd moments equal zero and the Fisher information matrix reduces to

\footnotetext{
${ }^{5}$ Dip test is implemented in the $\mathrm{R}$ package diptest.
} 


$$
j(\theta)=\left(\begin{array}{cccc}
\frac{\iota_{2}(\theta)}{\iota_{0}(\theta)} & 0 & \frac{1}{\sigma} & 0 \\
0 & \frac{\iota_{0}(\theta) \iota_{4}(\theta)-\iota_{2}(\theta)^{2}}{4 \iota_{0}(\theta)^{2}} & 0 & \frac{1}{\sigma} \frac{\iota_{2}(\theta)}{\iota_{0}(\theta)} \\
\frac{1}{\sigma} & 0 & -\frac{1}{\sigma^{2}}\left(\beta-\frac{\iota_{2}(\theta)}{\iota_{0}(\theta)}\right) & 0 \\
0 & \frac{1}{\sigma} \frac{\iota_{2}(\theta)}{\iota_{0}(\theta)} & 0 & -\frac{1}{\sigma^{2}}\left(1+3 \beta \frac{\iota_{2}(\theta)}{\iota_{0}(\theta)}+5 \frac{\iota_{4}(\theta)}{\iota_{0}(\theta)}\right)
\end{array}\right)
$$

where $\theta=(0, \beta, \lambda, \sigma)^{T}$; by inversion we get the variance for $\beta, j(\theta)_{\beta}^{-1}$ :

$$
\frac{4 \iota_{0}(\theta)^{2}\left(3 \beta \iota_{2}(\theta)+\iota_{0}(\theta)-5 \iota_{4}(\theta)\right)}{\iota_{0}(\theta)\left(3 \beta \iota_{4}(\theta) \iota_{2}(\theta)+3 \iota_{2}(\theta)^{2}-5 \iota_{4}(\theta)^{2}\right)+\iota_{2}(\theta)^{2}\left(5 \iota_{4}(\theta)-3 \beta \iota_{2}(\theta)\right)+\iota_{4}(\theta) \iota_{0}(\theta)^{2}},
$$

which for $\beta=0$ is positive finite constant, and the test statistic of the delta-test

$$
\sqrt{n} \frac{-\hat{\beta}}{3 \sqrt{\hat{j}(\hat{\theta})_{\beta}^{-1}}}
$$

reveals the cause for the low power of the delta-test for $\alpha=0$ even for the sample size of $n=10^{4}$, since the test statistics (28) rises linearly for beta close to zero. The slow rise of Cardan's discriminant (7) with respect to $\alpha$ or $\beta$ being close to zero shows another insight into the area of the delta-test with insufficient power to reject the null hypothesis (compare Figure 1 and Figure 2).

\begin{tabular}{rcccccclll}
\hline$\beta$ & -0.7 & -0.6 & -0.5 & -0.4 & -0.3 & -0.2 & -0.1 & 0 \\
\hline$\delta$-test & 1 & 0.98 & 0.82 & 0.46 & 0.12 & $\mathbf{0 . 0 1}$ & $\mathbf{0}$ & 0 \\
$\beta$-test & 1 & 1 & 1 & 1 & 0.99 & 0.84 & 0.39 & 0.04 \\
$l r$-test & 1 & 1 & 1 & 1 & 1 & 0.91 & 0.55 & $\mathbf{0 . 1 4}$ \\
\hline
\end{tabular}

Tab. 2. Power or size (in italics) of delta, beta and likelihood ratio test for $\alpha=0$, sample size $10^{4}$, and $10^{3}$ replications.

The insufficient power of delta-test estimated from the sample size with a length of $10^{3}$ disqualifies the delta-test to reject null hypothesis of bimodality even for negative $\beta$ significantly far from zero. The sample size with a length of $10^{4}$ raises power of the delta-test in rejecting the null hypothesis for $\beta<0$ (compare Table 9 and Table 10 ). This insufficient power of the delta-test can be overcome by using the beta or likelihood ratio test, where the variance is not influenced by the convergence of $\beta$ to zero (see Figure 2 and Table 2). However, the likelihood ratio test is biased for $\alpha \rightarrow 0$, since the influence of this parameter on the value of Cardan's discriminant is symmetric with respect to the line $\alpha=0$ (see Figure 1) and is increased with the growing variance of estimate $j(\theta)_{\alpha}^{-1}$. This results in a p-value of the likelihood ratio test higher than the significance level for $\alpha$ in the neighborhood of zero even when the null hypothesis holds (Tables 2, 5 and 6). At $\alpha=0$ size of this bias can be assessed through limiting the 


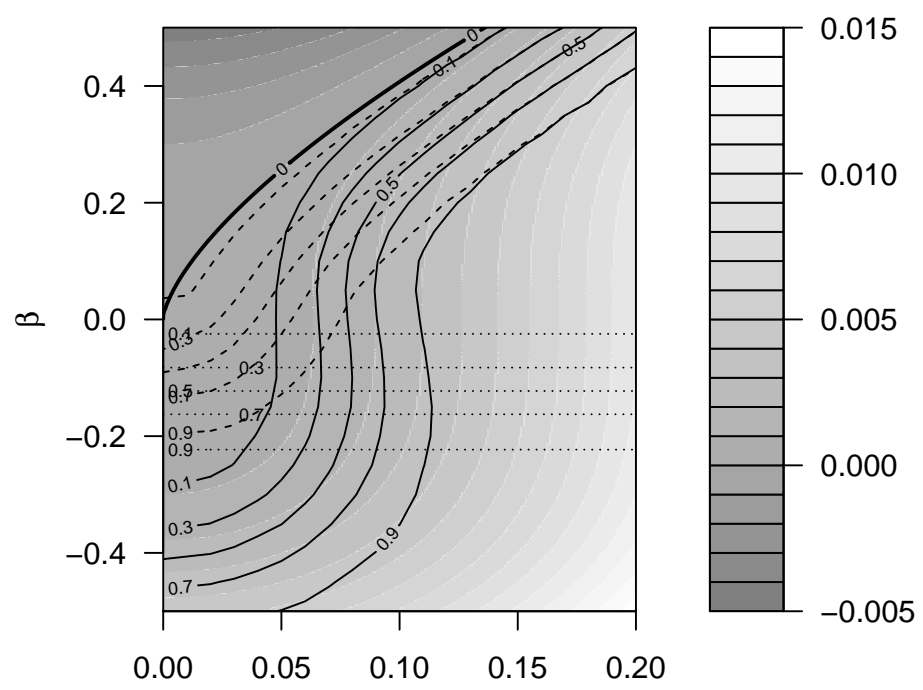

$\alpha$

Fig. 2. Contour plots of the level of Cardan's discriminant and power of delta (full line), beta (doted) and likelihood ratio (dashed) tests for a sample size of $10^{4}$ for $\alpha \in(0,0.2)$ and $\beta \in(-1,0)$. The thick line emphasizes the combination of alpha and $\beta$ for which Cardan's discriminant equals zero.

power of the likelihood ratio test by the power of the beta-test, since both of these tests are asymptotically equivalent.

Finally, the delta-test of unimodality with $H_{0}: \delta_{C} \geq 0$ and $H_{1}: \delta_{C}<0$ is compared with the dip test of unimodality and likelihood ratio test of unimodality given by switch of the inequality (24). According to expectation, the power of the dip test is lower than power of the delta-test specifically designed for cusp distribution when rejecting unimodality for various combinations of parameters (see Table 3 and Table 44. The likelihood ratio test performs even better than the delta-test especially for the smaller sample size and $\alpha$ closer to zero.

\section{CONCLUSION}

This paper is a contribution to the estimation and statistical testing of the stochastic cusp model. Reduction of the parametric space by two dimensions is shown, together with the connection of the maximum likelihood method to the method of moments. Secondly, it proposes a statistical test for bimodality of the stochastic cusp model using Cardan's discriminant, the delta method, and the maximum likelihood estimation. At the same time, it proposes a simpler test derived from a necessary condition on parameter 


\begin{tabular}{|c|c|c|c|c|c|c|c|c|c|c|c|}
\hline$\alpha$ & $\beta$ & 0.1 & 0.2 & 0.3 & 0.4 & 0.6 & 0.6 & 0.7 & 0.8 & 0.9 & 1 \\
\hline \multirow{3}{*}{0} & $l r$-test & 0.02 & 0.06 & 0.13 & 0.26 & 0.47 & 0.66 & 0.8 & 0.92 & 0.99 & 1 \\
\hline & $\delta$-test & 0 & 0 & 0 & 0.02 & 0.06 & 0.18 & 0.4 & 0.68 & 0.89 & 0.98 \\
\hline & $d i p$-test & 0 & 0 & 0 & 0.01 & 0.02 & 0.05 & 0.08 & 0.14 & 0.27 & 0.43 \\
\hline \multirow{3}{*}{0.1} & $l r$-test & 0 & 0.01 & 0.02 & 0.05 & 0.13 & 0.23 & 0.42 & 0.63 & 0.79 & 92 \\
\hline & $\delta$-test & 0 & 0 & 0 & 0.01 & 0.04 & 0.1 & 0.24 & 0.47 & 0.69 & 0.87 \\
\hline & dip-test & 0 & 0 & 0 & 0 & 0.01 & 0.02 & 0.04 & 0.07 & 0.12 & 0.2 \\
\hline \multirow{3}{*}{0.2} & $l r$-test & 0 & 0 & 0 & 0 & 0.01 & 0.03 & 0.08 & 0.21 & 0.41 & 0.64 \\
\hline & $\delta$-test & 0 & 0 & 0 & 0 & 0 & 0.02 & 0.06 & 0.16 & 0.33 & 0.56 \\
\hline & dip-test & 0 & 0 & 0 & 0 & 0 & 0 & 0 & 0.01 & 0.01 & 0.02 \\
\hline
\end{tabular}

Tab. 3. Power or size (in italics) of the inverse delta, likelihood ratio and the dip tests of unimodality, sample size $10^{3}$, and $10^{3}$ replications.

\begin{tabular}{|c|c|c|c|c|c|c|c|c|c|c|c|}
\hline$\alpha$ & & $\beta \quad 0.1$ & 0.2 & 0.3 & 0.4 & 0.6 & 0.6 & 0.7 & 0.8 & 0.9 & 1 \\
\hline \multirow{3}{*}{0} & $l r$-test & 0.05 & 0.2 & 0.76 & 0.97 & 1 & 1 & 1 & 1 & 1 & 1 \\
\hline & $\delta$-test & 0 & 0.04 & 0.42 & 0.91 & 1 & 1 & 1 & 1 & 1 & 1 \\
\hline & dip-test & 0 & 0 & 0.01 & 0.03 & 0.1 & 0.36 & 0.7 & 0.95 & 1 & 1 \\
\hline \multirow{3}{*}{0.1} & $l r$-test & 0 & 0 & 0 & 0.04 & 0.36 & 0.85 & 0.99 & 1 & 1 & 1 \\
\hline & $\delta$-test & 0 & 0 & 0 & 0.03 & 0.34 & 0.84 & 0.99 & 1 & 1 & 1 \\
\hline & dip-test & 0 & 0 & 0 & 0 & 0 & 0.01 & 0.06 & 0.22 & 0.56 & 0.89 \\
\hline \multirow{3}{*}{0.2} & $l r$-test & 0 & 0 & 0 & 0 & 0 & 0.01 & 0.21 & 0.81 & 1 & 1 \\
\hline & $\delta$-test & 0 & 0 & 0 & 0 & 0 & 0.01 & 0.19 & 0.79 & 1 & 1 \\
\hline & $d i p$-test & 0 & 0 & 0 & 0 & 0 & 0 & 0 & 0 & 0.02 & 0.11 \\
\hline
\end{tabular}

Tab. 4. Power or size (in italics) of the inverse delta, likelihood ratio and the dip tests of unimodality, sample size $10^{4}$, and $10^{3}$ replications.

beta for bimodality, which can be used for rejecting the bimodality as well. A simulation study comparing proposed tests and the likelihood ratio test shows that the test of parameter beta has a higher power than the test of Cardan's discriminant for the value of parameter $\alpha$ close to zero. A combination of these two tests with the one sided likelihood ratio test for Cardan's discriminant allows us to reject the bimodality hypothesis for various combinations of parameters $\alpha$ and $\beta$, however one should keep in mind that the one sided likelihood ratio test may reject the bimodality even when it holds. That is not the case for testing unimodality which is possible by the likelihood ratio test and the delta-test with reversed hypothesis and general test for the unimodality the dip test. The power to reject unimodality of the likelihood ratio test was found higher than the power of the delta-test especially for smaller sample size and parameter of asymmetry closer to zero. Both proposed tests have then outperformed the dip test in terms of power to reject the unimodality. 


\section{ACKNOWLEDGEMENT}

Support from the Czech Science Foundation by Grant No. P402/12/G097 is gratefully acknowledged.

(Received July 17, 2016)

\section{REFERENCES}

[1] V. I. Arnold: Catastrophe Theory. Springer-Verlag, Berlin 1992. DOI:10.1007/978-3-64258124-3

[2] J. Barunik and J. Kukačka: Realizing stock market crashes: stochastic cusp catastrophe model of returns under time-varying volatility. Quantitative Finance 15 (2015), 959-973. DOI:10.1080/14697688.2014.950319

[3] J. Barunik and M. Vošvrda: Can a stochastic cusp catastrophe model explain stock market crashes? J. Economic Dynamics Control 33 (2009), 1824-1836. DOI:10.1016/j.jedc.2009.04.004

[4] L. Cobb: Stochastic catastrophe models and multimodal distributions. Behavioral Sci. 23 (1978), 360-374. DOI:10.1002/bs.3830230407

[5] L. Cobb and B. Watson: Statistical catastrophe theory: An overview. Math. Modell. 1 (1980), 311-317. DOI:10.1016/0270-0255(80)90041-x

[6] L. Cobb: Parameter estimation for the cusp catastrophe model. Behavioral Sci. 26 (1981), 75-78. DOI:10.1002/bs.3830260107

[7] L. Cobb, P. Koppstein, and N.H. Chen: Estimation and moment recursion relations for multimodal distributions of the exponential family. J. Amer. Statist. Assoc. 78 (1983), 124-130. DOI:10.2307/2287118

[8] J. Creedy, J. Lye, and V. Martin: A non-linear model of the real US/UK exchange rate. Econom. Modell. 11 (1996), 669-686. DOI:10.1002/(sici)1099-1255(199611)11:6i669::aidjae415¿3.0.co;2-5

[9] C. Diks and J. Wang: Can a stochastic cusp catastrophe model explain housing market crashes? J. Econom. Dynamics Control 69 (2016), 68-88. DOI:10.1016/j.jedc.2016.05.008

[10] M. Fernandes: Financial crashes as endogenous jumps: estimation, testing and forecasting. J. Econom. Dynamics Control 30 (2006), 111-141. DOI:10.1016/j.jedc.2004.11.005

[11] R.P.P.P. Grasman, H.L.J. van der Maas, and E. J. Wagenmakers: Fitting the cusp catastrophe in R: A cusp package primer. J. Statist. Software 32 (2009), 1-28. DOI:10.18637/jss.v032.i08

[12] J. A. Hartigan and P. M. Hartigan: The dip test of unimodality. Ann. Statist. 13 (1985), 70-84. DOI:10.1214/aos/1176346577

[13] D. A. Kodde and F.C. Palm: Wald criteria for jointly testing equality and inequality restrictions. Econometrica 54 (1986), 1243-1248. DOI:10.2307/1912331

[14] S. K. Koh, W. M. Fong, and F. Chan: A Cardans discriminant approach to predicting currency crashes. J. Int. Money Finance 26 (2007), 131-148. DOI:10.1016/j.jimonfin.2006.08.001

[15] E. L. Lehman and J. P. Romano: Testing Statistical Hypotheses. Third edition. SpringerVerlag, New York 2005. DOI:10.1007/0-387-27605-x 
[16] A. W. Matz: Maximum likelihood parameter estimation for the quartic exponential distribution. Technometrics 20 (1978), 475-484. DOI:10.1080/00401706.1978.10489702

[17] R. Thom: Structural Stability and Morpohogenesis. W. A. Benjamin, New York 1975.

Jan Vořišsek, Institute of Information Theory and Automation, Department of Econometrics, The Czech Academy of Sciences, Pod Vodárenskou věži 4, 18208 Praha 8. Czech Republic.

e-mail: vorisek@karlin.mff.cuni.cz

\section{A. TABLES}

\begin{tabular}{lllllllllll}
\hline & 0 & 0.01 & 0.02 & 0.03 & 0.04 & 0.05 & 0.06 & 0.07 & 0.08 & 0.09 \\
\hline 0.35 & 0.02 & 0.01 & 0.01 & 0.01 & 0.02 & 0.02 & 0.03 & 0.03 & $\mathbf{0 . 0 4}$ & 0.05 \\
0.3 & 0.02 & 0.01 & 0.01 & 0.02 & 0.02 & 0.02 & 0.04 & $\mathbf{0 . 0 5}$ & 0.05 & 0.07 \\
0.25 & 0.03 & 0.03 & 0.03 & 0.03 & 0.03 & $\mathbf{0 . 0 4}$ & 0.06 & 0.06 & 0.08 & 0.1 \\
0.2 & 0.03 & 0.03 & 0.04 & 0.04 & $\mathbf{0 . 0 5}$ & 0.05 & 0.06 & 0.09 & 0.11 & 0.13 \\
0.15 & 0.05 & 0.04 & $\mathbf{0 . 0 6}$ & $\mathbf{0 . 0 4}$ & 0.06 & 0.07 & 0.1 & 0.11 & 0.16 & 0.16 \\
0.1 & $\mathbf{0 . 0 6}$ & $\mathbf{0 . 0 6}$ & 0.08 & 0.08 & 0.08 & 0.11 & 0.11 & 0.16 & 0.17 & 0.2 \\
0.05 & $\mathbf{0 . 0 8}$ & 0.1 & 0.11 & 0.1 & 0.11 & 0.14 & 0.16 & 0.17 & 0.2 & 0.24 \\
0 & $\mathbf{0 . 1 4}$ & 0.12 & 0.16 & 0.13 & 0.17 & 0.17 & 0.2 & 0.22 & 0.25 & 0.28 \\
-0.05 & 0.17 & 0.18 & 0.2 & 0.18 & 0.19 & 0.24 & 0.24 & 0.27 & 0.3 & 0.32 \\
\hline
\end{tabular}

Tab. 5. Power or the size (in italics) of the likelihood ratio test for $\alpha=0, \ldots, 0.09, \beta=-0.05, \ldots, 0.35,1000$ replications and sample size $10^{3}$. Bold font denotes the cases when the size of the test is higher than the significance level 0.05 .

\begin{tabular}{lllllllllll}
\hline & 0 & 0.01 & 0.02 & 0.03 & 0.04 & 0.05 & 0.06 & 0.07 & 0.08 & 0.09 \\
\hline 0.35 & 0 & 0 & 0 & 0 & 0 & 0 & 0.01 & 0.02 & 0.05 & 0.1 \\
0.3 & 0 & 0 & 0.01 & 0 & 0.01 & 0.01 & 0.03 & 0.08 & 0.16 & 0.22 \\
0.25 & 0.01 & 0.01 & 0.03 & 0.01 & 0.02 & 0.06 & 0.11 & 0.15 & 0.3 & 0.43 \\
0.2 & 0.02 & 0.03 & 0.03 & 0.04 & 0.07 & 0.13 & 0.24 & 0.37 & 0.47 & 0.63 \\
0.15 & $\mathbf{0 . 0 7}$ & 0.04 & $\mathbf{0 . 0 5}$ & 0.07 & 0.15 & 0.26 & 0.38 & 0.53 & 0.68 & 0.78 \\
0.1 & $\mathbf{0 . 0 8}$ & $\mathbf{0 . 0 6}$ & 0.08 & 0.16 & 0.27 & 0.4 & 0.55 & 0.7 & 0.83 & 0.9 \\
0.05 & $\mathbf{0 . 0 8}$ & 0.07 & 0.16 & 0.24 & 0.4 & 0.53 & 0.69 & 0.8 & 0.89 & 0.96 \\
0 & $\mathbf{0 . 1 4}$ & 0.19 & 0.28 & 0.4 & 0.52 & 0.65 & 0.78 & 0.88 & 0.93 & 0.97 \\
-0.05 & 0.3 & 0.35 & 0.44 & 0.52 & 0.65 & 0.77 & 0.85 & 0.92 & 0.96 & 0.99 \\
\hline
\end{tabular}

Tab. 6. Power or the size (in italics) of the likelihood ratio test for $\alpha=0, \ldots, 0.09, \beta=-0.05, \ldots, 0.35,1000$ replications and sample size $10^{4}$. Bold font denotes the cases when the size of the test is higher than the significance level 0.05 . 


\begin{tabular}{llllllllllll}
\hline & -0.5 & -0.4 & -0.3 & -0.2 & -0.1 & 0 & 0.1 & 0.2 & 0.3 & 0.4 & 0.5 \\
\hline 1 & 0.26 & 0.06 & 0 & 0 & 0 & 0 & 0 & 0 & 0 & 0.06 & 0.25 \\
0.9 & 0.43 & 0.13 & 0.02 & 0 & 0 & 0 & 0 & 0 & 0.02 & 0.15 & 0.44 \\
0.8 & 0.63 & 0.28 & 0.07 & 0 & 0 & 0 & 0 & 0 & 0.06 & 0.3 & 0.63 \\
0.7 & 0.77 & 0.44 & 0.13 & 0.02 & 0 & 0 & 0 & 0.02 & 0.15 & 0.44 & 0.78 \\
0.6 & 0.87 & 0.61 & 0.26 & $\mathbf{0 . 0 4}$ & 0 & 0 & 0 & 0.05 & 0.3 & 0.63 & 0.88 \\
0.5 & 0.94 & 0.79 & 0.4 & 0.08 & 0 & 0 & 0 & 0.1 & 0.42 & 0.76 & 0.95 \\
0.4 & 0.98 & 0.88 & 0.54 & 0.17 & $\mathbf{0 . 0 1}$ & 0 & $\mathbf{0 . 0 1}$ & 0.14 & 0.55 & 0.85 & 0.97 \\
0.3 & 0.99 & 0.93 & 0.64 & 0.19 & $\mathbf{0 . 0 1}$ & 0 & $\mathbf{0}$ & 0.18 & 0.64 & 0.91 & 0.99 \\
0.2 & 0.99 & 0.94 & 0.7 & 0.2 & $\mathbf{0 . 0 1}$ & 0 & $\mathbf{0 . 0 1}$ & 0.21 & 0.69 & 0.94 & 1 \\
0.1 & 0.99 & 0.95 & 0.66 & 0.19 & $\mathbf{0 . 0 2}$ & 0 & $\mathbf{0 . 0 1}$ & 0.21 & 0.67 & 0.95 & 0.99 \\
0 & 0.99 & 0.93 & 0.61 & 0.16 & $\mathbf{0 . 0 1}$ & 0 & $\mathbf{0 . 0 1}$ & 0.18 & 0.62 & 0.93 & 1 \\
-0.1 & 0.98 & 0.89 & 0.54 & 0.13 & $\mathbf{0 . 0 1}$ & $\mathbf{0}$ & $\mathbf{0 . 0 1}$ & 0.15 & 0.51 & 0.89 & 0.98 \\
-0.2 & 0.96 & 0.82 & 0.46 & 0.09 & $\mathbf{0 . 0 1}$ & $\mathbf{0}$ & $\mathbf{0 . 0 2}$ & 0.12 & 0.44 & 0.81 & 0.96 \\
-0.3 & 0.91 & 0.73 & 0.36 & 0.09 & $\mathbf{0 . 0 1}$ & $\mathbf{0}$ & $\mathbf{0}$ & 0.08 & 0.37 & 0.71 & 0.92 \\
-0.4 & 0.85 & 0.63 & 0.26 & 0.05 & $\mathbf{0 . 0 1}$ & $\mathbf{0}$ & $\mathbf{0}$ & 0.05 & 0.27 & 0.58 & 0.83 \\
-0.5 & 0.74 & 0.47 & 0.21 & $\mathbf{0 . 0 4}$ & $\mathbf{0}$ & $\mathbf{0}$ & $\mathbf{0}$ & $\mathbf{0 . 0 5}$ & 0.18 & 0.49 & 0.74 \\
-0.6 & 0.64 & 0.36 & 0.14 & $\mathbf{0 . 0 2}$ & $\mathbf{0}$ & $\mathbf{0}$ & $\mathbf{0}$ & $\mathbf{0 . 0 2}$ & 0.14 & 0.37 & 0.63 \\
-0.7 & 0.51 & 0.27 & 0.08 & $\mathbf{0 . 0 3}$ & $\mathbf{0}$ & $\mathbf{0}$ & $\mathbf{0 . 0 1}$ & $\mathbf{0 . 0 2}$ & 0.1 & 0.26 & 0.52 \\
-0.8 & 0.37 & 0.19 & 0.07 & $\mathbf{0 . 0 1}$ & $\mathbf{0}$ & $\mathbf{0}$ & $\mathbf{0}$ & $\mathbf{0 . 0 2}$ & 0.08 & 0.2 & 0.37 \\
-0.9 & 0.28 & 0.14 & $\mathbf{0 . 0 3}$ & $\mathbf{0 . 0 1}$ & $\mathbf{0}$ & $\mathbf{0}$ & $\mathbf{0}$ & $\mathbf{0 . 0 1}$ & 0.05 & 0.13 & 0.28 \\
-1 & 0.22 & 0.1 & $\mathbf{0 . 0 3}$ & $\mathbf{0 . 0 1}$ & $\mathbf{0}$ & $\mathbf{0}$ & $\mathbf{0}$ & $\mathbf{0}$ & $\mathbf{0 . 0 2}$ & 0.08 & 0.21 \\
\hline
\end{tabular}

Tab. 7. Power or the size (in italics) of the delta test for $\alpha=-0.5, \ldots, 0.5, \beta=-1, \ldots, 1$, sample size $10^{3}$, and $10^{3}$ replications. Bold font denotes the cases when the power of the test is lower than the significance level 0.05 . 


\begin{tabular}{llllllllllll}
\hline & -0.5 & -0.4 & -0.3 & -0.2 & -0.1 & 0 & 0.1 & 0.2 & 0.3 & 0.4 & 0.5 \\
\hline 1 & 0.96 & 0.12 & 0 & 0 & 0 & 0 & 0 & 0 & 0 & 0.12 & 0.97 \\
0.9 & 1 & 0.71 & 0.01 & 0 & 0 & 0 & 0 & 0 & 0 & 0.68 & 1 \\
0.8 & 1 & 0.99 & 0.18 & 0 & 0 & 0 & 0 & 0 & 0.18 & 0.98 & 1 \\
0.7 & 1 & 1 & 0.74 & 0 & 0 & 0 & 0 & 0.01 & 0.72 & 1 & 1 \\
0.6 & 1 & 1 & 0.99 & 0.18 & 0 & 0 & 0 & 0.14 & 0.98 & 1 & 1 \\
0.5 & 1 & 1 & 1 & 0.66 & 0 & 0 & 0 & 0.68 & 1 & 1 & 1 \\
0.4 & 1 & 1 & 1 & 0.96 & $\mathbf{0 . 0 4}$ & 0 & $\mathbf{0 . 0 5}$ & 0.96 & 1 & 1 & 1 \\
0.3 & 1 & 1 & 1 & 1 & 0.29 & 0 & 0.3 & 1 & 1 & 1 & 1 \\
0.2 & 1 & 1 & 1 & 1 & 0.67 & 0 & 0.68 & 1 & 1 & 1 & 1 \\
0.1 & 1 & 1 & 1 & 1 & 0.85 & 0 & 0.84 & 1 & 1 & 1 & 1 \\
0 & 1 & 1 & 1 & 1 & 0.85 & 0 & 0.84 & 1 & 1 & 1 & 1 \\
-0.1 & 1 & 1 & 1 & 1 & 0.78 & $\mathbf{0}$ & 0.77 & 1 & 1 & 1 & 1 \\
-0.2 & 1 & 1 & 1 & 1 & 0.8 & $\mathbf{0}$ & 0.79 & 1 & 1 & 1 & 1 \\
-0.3 & 1 & 1 & 1 & 1 & 0.85 & 0.12 & 0.88 & 1 & 1 & 1 & 1 \\
-0.4 & 1 & 1 & 1 & 1 & 0.94 & 0.47 & 0.94 & 1 & 1 & 1 & 1 \\
-0.5 & 1 & 1 & 1 & 1 & 0.99 & 0.82 & 0.98 & 1 & 1 & 1 & 1 \\
-0.6 & 1 & 1 & 1 & 1 & 1 & 0.98 & 1 & 1 & 1 & 1 & 1 \\
-0.7 & 1 & 1 & 1 & 1 & 1 & 1 & 1 & 1 & 1 & 1 & 1 \\
-0.8 & 1 & 1 & 1 & 1 & 1 & 1 & 1 & 1 & 1 & 1 & 1 \\
-0.9 & 1 & 1 & 1 & 1 & 1 & 1 & 1 & 1 & 1 & 1 & 1 \\
-1 & 1 & 1 & 1 & 1 & 1 & 1 & 1 & 1 & 1 & 1 & 1 \\
\hline
\end{tabular}

Tab. 8. Power or the size (in italics) of the delta test for $\alpha=-0.5, \ldots, 0.5, \beta=-1, \ldots, 1$, sample size $10^{4}$, and $10^{3}$ replications. Bold font denotes the cases when the power of the test is lower than the significance level 0.05 . 


\begin{tabular}{|c|c|c|c|c|c|c|c|c|c|c|c|}
\hline & 0.1 & 0.11 & 0.12 & 0.13 & 0.14 & 0.15 & 0.16 & 0.17 & 0.18 & 0.19 & 0.2 \\
\hline 1 & 0 & 0 & 0 & 0 & 0 & 0 & 0 & 0 & 0 & 0 & 0 \\
\hline 0.95 & 0 & 0 & 0 & 0 & 0 & 0 & 0 & 0 & 0 & 0 & 0 \\
\hline 0.9 & 0 & 0 & 0 & 0 & 0 & 0 & 0 & 0 & 0 & 0 & 0 \\
\hline 0.85 & 0 & 0 & 0 & 0 & 0 & 0 & 0 & 0 & 0 & 0 & 0 \\
\hline 0.8 & 0 & 0 & 0 & 0 & 0 & 0 & 0 & 0 & 0 & 0.01 & 0 \\
\hline 0.75 & 0 & 0 & 0 & 0 & 0 & 0 & 0 & 0 & 0.01 & 0.01 & 0.01 \\
\hline 0.7 & 0 & 0 & 0 & 0 & 0 & 0 & 0 & 0 & 0.01 & 0.01 & 0.02 \\
\hline 0.65 & 0 & 0 & 0 & 0 & 0.01 & 0 & 0.01 & 0.01 & 0.02 & 0.02 & 0.02 \\
\hline 0.6 & 0 & 0 & 0 & 0 & 0.01 & 0.01 & 0.03 & 0.01 & 0.02 & 0.04 & 0.05 \\
\hline 0.55 & 0 & 0 & 0.01 & 0.01 & 0.01 & 0.01 & 0.02 & 0.03 & 0.04 & 0.04 & 0.06 \\
\hline 0.5 & 0 & 0.01 & 0.01 & 0.01 & 0.01 & 0.02 & 0.03 & 0.03 & 0.06 & 0.07 & 0.07 \\
\hline 0.45 & 0.01 & 0.01 & 0.01 & 0.01 & 0.02 & 0.03 & 0.05 & 0.06 & 0.06 & 0.08 & 0.11 \\
\hline 0.4 & 0 & 0.01 & 0.01 & 0.02 & 0.02 & 0.04 & 0.06 & 0.06 & 0.09 & 0.12 & 0.15 \\
\hline 0.35 & 0 & 0.02 & 0.02 & 0.02 & 0.04 & 0.05 & 0.08 & 0.1 & 0.1 & 0.14 & 0.14 \\
\hline 0.3 & 0.01 & 0.01 & 0.02 & 0.03 & 0.03 & 0.06 & 0.06 & 0.1 & 0.13 & 0.16 & 0.18 \\
\hline 0.25 & 0.01 & 0.01 & 0.03 & 0.03 & 0.04 & 0.05 & 0.07 & 0.11 & 0.11 & 0.14 & 0.18 \\
\hline 0.2 & 0.01 & 0.02 & 0.02 & 0.03 & 0.04 & 0.06 & 0.09 & 0.1 & 0.14 & 0.16 & 0.21 \\
\hline 0.15 & 0.01 & 0.01 & 0.03 & 0.04 & 0.06 & 0.07 & 0.06 & 0.12 & 0.13 & 0.16 & 0.2 \\
\hline 0.1 & 0.01 & 0.02 & 0.02 & 0.04 & 0.04 & 0.07 & 0.08 & 0.11 & 0.14 & 0.14 & 0.2 \\
\hline 0.05 & 0.02 & 0.02 & 0.02 & 0.04 & 0.05 & 0.06 & 0.08 & 0.09 & 0.14 & 0.15 & 0.17 \\
\hline 0 & 0.01 & 0.02 & 0.02 & 0.03 & 0.04 & 0.06 & 0.07 & 0.11 & 0.12 & 0.16 & 0.17 \\
\hline-0.05 & 0.01 & 0.01 & 0.02 & 0.03 & 0.05 & 0.05 & 0.07 & 0.1 & 0.09 & 0.14 & 0.15 \\
\hline-0.1 & 0.01 & 0.02 & 0.02 & 0.03 & 0.04 & 0.04 & 0.06 & 0.08 & 0.08 & 0.13 & 0.14 \\
\hline-0.15 & 0.01 & 0.01 & 0.02 & 0.02 & 0.04 & 0.04 & 0.04 & 0.07 & 0.09 & 0.1 & 0.12 \\
\hline-0.2 & 0.01 & 0.01 & 0.02 & 0.02 & 0.03 & 0.04 & 0.05 & 0.06 & 0.08 & 0.09 & 0.09 \\
\hline-0.25 & 0.01 & 0.01 & 0.02 & 0.02 & 0.03 & 0.04 & 0.04 & 0.05 & 0.07 & 0.07 & 0.1 \\
\hline-0.3 & 0 & 0.01 & 0.01 & 0.02 & 0.02 & 0.02 & 0.04 & 0.05 & 0.06 & 0.08 & 0.08 \\
\hline-0.35 & 0.01 & 0.01 & 0.01 & 0.02 & 0.02 & 0.03 & 0.03 & 0.03 & 0.04 & 0.06 & 0.09 \\
\hline-0.4 & 0 & 0.01 & 0.01 & 0.02 & 0.01 & 0.02 & 0.03 & 0.02 & 0.03 & 0.06 & 0.06 \\
\hline-0.45 & 0 & 0 & 0.01 & 0.01 & 0.02 & 0.02 & 0.02 & 0.02 & 0.05 & 0.05 & 0.04 \\
\hline-0.5 & 0 & 0.01 & 0.01 & 0.01 & 0.01 & 0.01 & 0.02 & 0.02 & 0.04 & 0.03 & 0.05 \\
\hline-0.55 & 0 & 0.01 & 0.01 & 0 & 0.01 & 0.01 & 0.01 & 0.02 & 0.02 & 0.04 & 0.04 \\
\hline-0.6 & 0 & 0 & 0.01 & 0 & 0.01 & 0 & 0.02 & 0.02 & 0.01 & 0.02 & 0.03 \\
\hline-0.65 & 0 & 0 & 0 & 0.01 & 0.01 & 0.01 & 0.01 & 0.02 & 0.02 & 0.02 & 0.02 \\
\hline-0.7 & 0 & 0 & 0 & 0 & 0.01 & 0.01 & 0.01 & 0.01 & 0.02 & 0.02 & 0.02 \\
\hline-0.75 & 0 & 0.01 & 0 & 0 & 0 & 0.01 & 0.01 & 0.01 & 0.02 & 0.01 & 0.02 \\
\hline-0.8 & 0 & 0 & 0 & 0 & 0 & 0 & 0.01 & 0.01 & 0.01 & 0.01 & 0.02 \\
\hline-0.85 & 0 & 0 & 0 & 0 & 0 & 0 & 0.01 & 0.01 & 0.01 & 0.01 & 0 \\
\hline-0.9 & 0 & 0 & 0 & 0 & 0 & 0 & 0 & 0.01 & 0 & 0.01 & 0.02 \\
\hline-0.95 & 0 & 0 & 0 & 0 & 0 & 0 & 0 & 0 & 0 & 0.01 & 0 \\
\hline-1 & 0 & 0 & 0 & 0 & 0 & 0 & 0 & 0.01 & 0.01 & 0 & 0.01 \\
\hline
\end{tabular}

Tab. 9. Power or the size (in italics) of the delta test for $\alpha=0.1, \ldots, 0.2, \beta=-1, \ldots, 1$, sample size $10^{3}$, and $10^{3}$ replications. Bold font denotes the cases when the power of the test is lower than the significance level 0.05 . 


\begin{tabular}{|c|c|c|c|c|c|c|c|c|c|c|c|}
\hline & 0 & 0.01 & 0.02 & 0.03 & 0.04 & 0.05 & 0.06 & 0.07 & 0.08 & 0.09 & 0.1 \\
\hline 1 & 0 & 0 & 0 & 0 & 0 & 0 & 0 & 0 & 0 & 0 & 0 \\
\hline 0.95 & 0 & 0 & 0 & 0 & 0 & 0 & 0 & 0 & 0 & 0 & 0 \\
\hline 0.9 & 0 & 0 & 0 & 0 & 0 & 0 & 0 & 0 & 0 & 0 & 0 \\
\hline 0.85 & 0 & 0 & 0 & 0 & 0 & 0 & 0 & 0 & 0 & 0 & 0 \\
\hline 0.8 & 0 & 0 & 0 & 0 & 0 & 0 & 0 & 0 & 0 & 0 & 0 \\
\hline 0.75 & 0 & 0 & 0 & 0 & 0 & 0 & 0 & 0 & 0 & 0 & 0 \\
\hline 0.7 & 0 & 0 & 0 & 0 & 0 & 0 & 0 & 0 & 0 & 0 & 0 \\
\hline 0.65 & 0 & 0 & 0 & 0 & 0 & 0 & 0 & 0 & 0 & 0 & 0 \\
\hline 0.6 & 0 & 0 & 0 & 0 & 0 & 0 & 0 & 0 & 0 & 0 & 0 \\
\hline 0.55 & 0 & 0 & 0 & 0 & 0 & 0 & 0 & 0 & 0 & 0 & 0 \\
\hline 0.5 & 0 & 0 & 0 & 0 & 0 & 0 & 0 & 0 & 0 & 0 & 0 \\
\hline 0.45 & 0 & 0 & 0 & 0 & 0 & 0 & 0 & 0 & 0 & 0 & 0.01 \\
\hline 0.4 & 0 & 0 & 0 & 0 & 0 & 0 & 0 & 0 & 0.01 & 0.02 & 0.05 \\
\hline 0.35 & 0 & 0 & 0 & 0 & 0 & 0 & 0 & 0.02 & 0.04 & 0.06 & 0.15 \\
\hline 0.3 & 0 & 0 & 0 & 0 & 0 & 0.01 & 0.03 & 0.05 & 0.09 & 0.18 & 0.3 \\
\hline 0.25 & 0 & 0 & 0 & 0 & 0.01 & 0.02 & 0.05 & 0.11 & 0.22 & 0.34 & 0.48 \\
\hline 0.2 & 0 & 0 & 0 & 0 & 0.01 & 0.05 & 0.1 & 0.22 & 0.34 & 0.5 & 0.65 \\
\hline 0.15 & 0 & 0 & 0 & 0.01 & 0.03 & 0.08 & 0.15 & 0.28 & 0.47 & 0.61 & 0.78 \\
\hline 0.1 & 0 & 0 & 0 & 0.01 & 0.05 & 0.1 & 0.21 & 0.36 & 0.54 & 0.7 & 0.84 \\
\hline 0.05 & 0 & 0 & 0.01 & 0.01 & 0.06 & 0.12 & 0.22 & 0.35 & 0.53 & 0.72 & 0.85 \\
\hline 0 & 0 & 0 & 0 & 0.02 & 0.06 & 0.11 & 0.23 & 0.36 & 0.54 & 0.68 & 0.81 \\
\hline-0.05 & 0 & 0 & 0.01 & 0.01 & 0.06 & 0.12 & 0.2 & 0.37 & 0.52 & 0.67 & 0.82 \\
\hline-0.1 & 0 & 0 & 0.01 & 0.03 & 0.05 & 0.12 & 0.22 & 0.35 & 0.5 & 0.67 & 0.78 \\
\hline-0.15 & 0 & 0.01 & 0.01 & 0.04 & 0.07 & 0.12 & 0.25 & 0.37 & 0.52 & 0.65 & 0.77 \\
\hline-0.2 & 0.01 & 0.01 & 0.03 & 0.05 & 0.09 & 0.17 & 0.26 & 0.42 & 0.51 & 0.67 & 0.78 \\
\hline-0.25 & 0.04 & 0.05 & 0.07 & 0.1 & 0.17 & 0.24 & 0.38 & 0.44 & 0.61 & 0.71 & 0.82 \\
\hline-0.3 & 0.11 & 0.11 & 0.13 & 0.19 & 0.27 & 0.38 & 0.46 & 0.57 & 0.69 & 0.77 & 0.86 \\
\hline-0.35 & 0.24 & 0.26 & 0.3 & 0.33 & 0.41 & 0.5 & 0.6 & 0.71 & 0.77 & 0.84 & 0.9 \\
\hline-0.4 & 0.45 & 0.46 & 0.48 & 0.56 & 0.61 & 0.64 & 0.74 & 0.82 & 0.85 & 0.91 & 0.94 \\
\hline-0.45 & 0.65 & 0.66 & 0.69 & 0.73 & 0.78 & 0.82 & 0.86 & 0.88 & 0.91 & 0.95 & 0.98 \\
\hline-0.5 & 0.85 & 0.83 & 0.85 & 0.88 & 0.89 & 0.9 & 0.95 & 0.95 & 0.97 & 0.97 & 0.98 \\
\hline-0.55 & 0.94 & 0.93 & 0.93 & 0.93 & 0.96 & 0.95 & 0.97 & 0.98 & 0.98 & 0.99 & 1 \\
\hline-0.6 & 0.97 & 0.98 & 0.98 & 0.99 & 0.99 & 0.98 & 0.99 & 0.99 & 0.99 & 1 & 1 \\
\hline-0.65 & 1 & 0.99 & 1 & 0.99 & 1 & 1 & 1 & 1 & 1 & 1 & 1 \\
\hline-0.7 & 1 & 1 & 1 & 1 & 1 & 1 & 1 & 1 & 1 & 1 & 1 \\
\hline-0.75 & 1 & 1 & 1 & 1 & 1 & 1 & 1 & 1 & 1 & 1 & 1 \\
\hline-0.8 & 1 & 1 & 1 & 1 & 1 & 1 & 1 & 1 & 1 & 1 & 1 \\
\hline-0.85 & 1 & 1 & 1 & 1 & 1 & 1 & 1 & 1 & 1 & 1 & 1 \\
\hline-0.9 & 1 & 1 & 1 & 1 & 1 & 1 & 1 & 1 & 1 & 1 & 1 \\
\hline-0.95 & 1 & 1 & 1 & 1 & 1 & 1 & 1 & 1 & 1 & 1 & 1 \\
\hline-1 & 1 & 1 & 1 & 1 & 1 & 1 & 1 & 1 & 1 & 1 & 1 \\
\hline
\end{tabular}

Tab. 10. Power or the size (in italics) of the delta test for $\alpha=0, \ldots, 0.1, \beta=-1, \ldots, 1$, sample size $10^{4}$, and $10^{3}$ replications.

Bold font denotes the cases when the power of the test is lower than the significance level 0.05 . 